\title{
Fourier mode dynamics for the nonlinear Schrödinger equation in one-dimensional bounded domains
}

\author{
J. G. Caputo ${ }^{1}$, N. K. Efremidis ${ }^{2}$, Chao Hang ${ }^{3,4}$ \\ ${ }^{1}$ Laboratoire de Mathématiques, INSA de Rouen, \\ B. P.8, 76801 Saint-Etienne du Rouvray, France \\ E-mail: caputo@insa-rouen.fr \\ ${ }^{2}$ Department of applied Mathematics, \\ University of Crete, 71409 Heraklion, Crete, Greece \\ E-mail: nefrem@tem.uoc.gr \\ ${ }^{3}$ Centro de Física Teórica e Computacional, \\ Faculdade de Ciências, Universidade de Lisboa, \\ Avenida Professor Gama Pinto 2, \\ Lisboa 1649-003, Portugal \\ E-mail: chang@cii.fc.ul.pt \\ ${ }^{4}$ Department of Physics and State Key Laboratory of Precision Spectroscopy, \\ East China Normal University, \\ Shanghai 200062, China \\ (Dated: September 26, 2018)
}

\begin{abstract}
We analyze the 1D focusing nonlinear Schrödinger equation in a finite interval with homogeneous Dirichlet or Neumann boundary conditions. There are two main dynamics, the collapse which is very fast and a slow cascade of Fourier modes. For the cubic nonlinearity the calculations show no long term energy exchange between Fourier modes as opposed to higher nonlinearities. This slow dynamics is explained by fairly simple amplitude equations for the resonant Fourier modes. Their solutions are well behaved so filtering high frequencies prevents collapse. Finally these equations elucidate the unique role of the zero mode for the Neumann boundary conditions.
\end{abstract}

\section{INTRODUCTION}

The nonlinear Schrödinger equation in 2 or 3 spatial dimensions with a focusing nonlinearity has been studied intensively because of the collapse phenomenon, see for example the reviews of Berge [1] and Sulem [2]. Most of these studies have been done for an infinite domain, for which the equation is invariant by a scaling transformation. This symmetry is important to determine the conditions for collapse. In many applications like for example a laser propagating in an optical fiber, the domain is finite so the boundaries play an important role. A pioneering study was conducted by Fibich and Merle [3] for the $2 \mathrm{D}$ cubic nonlinear Schrödinger equation. They showed that circularly symmetric ground state waveguide solutions are stable and that the critical power condition for collapse is sharp unlike for an infinite domain. For small amplitudes the ground states reduce to the Bessel linear modes of the Laplacian. An interesting problem is then how these modes exchange energy as the solution evolves. This issue is important since some of these modes can be filtered out. More generally this energy exchange between linear modes is related to the old problem studied by Fermi-Pasta and Ulam (see the first section of [4] for subsequent developments). For a chain of oscillators with cubic nonlinearities, a medium amplitude Fourier mode initial condition gives rise to a cascade of higher Fourier modes and energy flows back into the initial mode. For the cubic nonlinear Schrödinger equation in $1 \mathrm{D}$ we expect a similar recurrence because the underlying long wave reduction model remains the Korteweg-de
Vries equation. This recurrence is different from what happens in turbulence where there is a one way flow of energy in wave numbers. For example Muraki [5] studied this one-way cascade for the Burgers equation. Using the Cole-Hopf transformation, he was able to quantify the phenomenon. Note finally the study [6] by Zakharov et al of wave turbulence carried out on a $1 \mathrm{D}$ model in Fourier space.

Following a similar approach, in this article we have analyzed the 1D nonlinear Schrödinger equation with cubic and quintic nonlinearities on a finite interval with Dirichlet or Neumann boundary conditions. We have chosen to work in $1 \mathrm{D}$ to benefit from the Fourier machinery which enables to solve the problem fairly easily. A relatively small number of Fourier modes are necessary to describe well the solution when it is not singular. Another advantage is that the analysis can be done easier than for the $2 \mathrm{D}$ case. We have studied the stationary solutions and their stability. We have also obtained simple models for the resonant transfer of energy between Fourier modes for the cubic and quintic nonlinearities. As expected there is no transfer for the cubic nonlinearity reflecting the integrability of the equation. For a quintic nonlinearity a resonant transfer exists. The solutions of these reduced models compare well to the numerical solutions of the partial differential equation. This method of analysis of the resonant transfers of energy can be extended to higher dimensions. The principle remains the same but of course the machinery will be much more complicated. From another point of view our study presents the time evolution of a solution prior to collapse. Finally we show that filtering prevents collapse. 
The article is organized as follows. In section II we review the conservation laws and the Virial identity, the main theoretical tool, for both the Dirichlet and the Neumann boundary conditions. Section III presents the ground states and their stability. Numerical results are shown in section IV and explained using a new model of resonant energy transfer in section V. We conclude in section VI.

\section{CONSERVED QUANTITIES AND VIRIAL RELATIONS}

We consider the one dimensional nonlinear Schrödinger (NLS) equation

$$
i \psi_{t}+\psi_{x x}+|\psi|^{2 d} \psi=0
$$

on a smooth, bounded domain $[0, \pi]$ with the homogeneous Dirichlet boundary condition

$$
\psi(x=0)=\psi(x=\pi)=0,
$$

or the Neumann boundary condition

$$
\psi_{x}(x=0)=\psi_{x}(x=\pi)=0 .
$$

Here $d$ is a positive integer with $d=1,2$, and 3 corresponding to the cubic, quintic, and septic nonlinearities.

Eq. (1) with both Dirichlet and Neumann boundary conditions admits the following conserved quantities, the $\mathrm{L}^{2}$ norm

$$
P=\int_{0}^{\pi}|\psi|^{2} d x
$$

which is the total power in optics and the Hamiltonian

$$
H=\int_{0}^{\pi}\left(\left|\psi_{x}\right|^{2}-\frac{1}{d+1}|\psi|^{2 d+2}\right) d x .
$$

The momentum

$$
\Pi=i \int_{0}^{\pi}\left(\psi \psi_{x}^{*}-\psi^{*} \psi_{x}\right) d x
$$

which is conserved for the infinite domain now has a flux

$$
\Pi_{t}=-4\left[\left|\psi_{x}\right|^{2}\right]_{0}^{\pi},
$$

for Dirichlet boundary condition and

$$
\Pi_{t}=\left[\left(|\psi|^{2}\right)_{x x}+\frac{2 d}{d+1}|\psi|^{2 d+2}\right]_{0}^{\pi},
$$

for Neumann boundary condition.

We can analyze the evolution of following integral quantities related with the model (1)

$$
\begin{aligned}
I_{1}(t) & =\int_{0}^{\pi}|\psi|^{2} x^{2} d x \\
I_{2}(t) & =\int_{0}^{\pi}\left(\left|\psi_{x}\right|^{2}-\frac{d}{2(d+1)}|\psi|^{2 d+2}\right) d x \\
& =H-\frac{1}{2} \int_{0}^{\pi} \frac{d-2}{d+1}|\psi|^{2 d+2} d x
\end{aligned}
$$

Here, $I_{1}$ is the variance, which is a common tool for predicting collapse of NLS equation solutions in infinite domain and we assume that $I_{j}(j=1,2)$ are initially well defined.

For Dirichlet boundary, some algebra leads to

$$
\frac{d^{2} I_{1}}{d t^{2}}=-4 \pi\left[\left|\psi_{x}\right|^{2}\right]_{x=\pi}+8 H-4 \int_{0}^{\pi} \frac{d-2}{d+1}|\psi|^{2 d+2} d x
$$

which shows that $H<0$ is a sufficient condition for collapse. Notice that the right hand side of (10) can be negative even though $H>0$. In the infinite domain the first term is absent. Therefore, compared with the infinite line, Dirichlet boundaries focus the solution and enhance the collapse.

For the Neumann boundary conditions, we have

$$
\begin{aligned}
\frac{d^{2} I_{1}}{d t^{2}} & =2 \pi\left[\left(|\psi|^{2}\right)_{x x}+\frac{2 d}{d+1}|\psi|^{2 d+2}\right]_{x=\pi} \\
& +8 H-4 \int_{0}^{\pi} \frac{d-2}{d+1}|\psi|^{2 d+2} d x
\end{aligned}
$$

From Eq. (11) we notice that $H<0$ is not a sufficient condition for the collapse. Therefore the Neumann boundary can be either reflecting or absorbing and enhance or suppress the collapse.

\section{BOUND STATES}

The time periodic solutions of Eq. (1) can be searched in the form

$$
\psi(z, x)=u(x) \exp (i E t)
$$

where $u(x)$ is a real function and $E$ is the propagation constant which is also real. The resulting equation then reads

$$
-E u+u^{\prime \prime}+u^{2 d+1}=0
$$

with the Dirichlet boundary conditions

$$
u(x=0)=u(x=\pi)=0
$$

and the Neumann boundary conditions

$$
u_{x}(x=0)=u_{x}(x=\pi)=0 .
$$

A conserved quantity of Eq. (13) can be found by multiplying with $u^{\prime}(x)$ and integrating over $x$

$$
K=\frac{1}{2}\left(u^{\prime}\right)^{2}-\frac{E}{2} u^{2}+\frac{1}{2 d+2} u^{2 d+2},
$$

which can be further integrated to give

$$
\int d x= \pm \frac{1}{2} \int d z\left[z\left(4 K+2 E z-\frac{2}{d+1} z^{d+1}\right)\right]^{-1 / 2}
$$




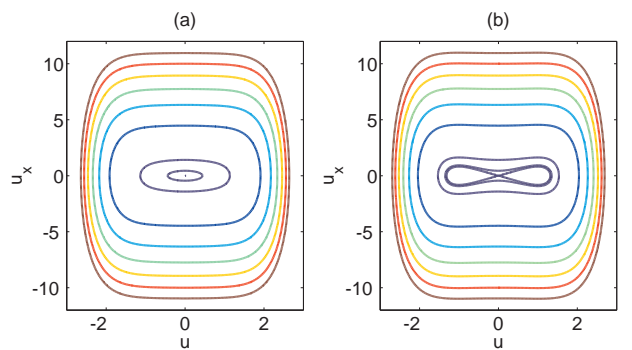

FIG. 1: Phase portrait associated with Eq. (16) for $E=-1$ (left panel) and $E=1$ (right panel) for $d=2$. The levels presented for $K$ are $K=0,0.1,1,10,20,30,40,50,60$.

by defining $z=u^{2}$. The above integral can be expressed in terms of Jacobi elliptic functions in both the cases of cubic and quintic nonlinearities. However, in the latter case the resulting expressions are rather complicated.

The phase portrait associated with Eq. (16) is shown in Fig. 1 for $E=-1$ (left panel) and $E=1$ (right panel). The points at $u=0\left(u_{x}=0\right)$ correspond to the solutions on the boundaries for Dirichlet (Neumann) case.

If $u \ll 1$, the nonlinearity does not play an important role and the solutions are close to the linear limit of Eq. (13), i.e.

$$
u_{m}(x)=A \sin (m x),
$$

for Dirichlet boundary condition and

$$
u_{m}(x)=A \cos (m x),
$$

for Neumann boundary condition. Here $m$ denotes the index of the wave number $m=0,1,2, \cdots$ and $A$ denotes the amplitude with $A \ll 1$. The propagation constant, $K$, and the power are then given by

$$
E_{m}=-m^{2}, \quad K_{m}=\frac{m^{2}}{2} A^{2}, \quad P=\frac{\pi}{2} A^{2} .
$$

When $|u|$ starts to increase, the propagation constant, as well as the form of the solutions, is slightly modified due to the effect of nonlinearity, i.e. $E=E_{m}+\delta E$ with $\delta E \ll E_{m}$. The relationship between the correction of the propagation constant $\delta E$ and amplitude $A$ (and thus $P)$ can be computed by

$$
\delta E=\frac{(2 d+2) !}{2^{2 d+1}[(d+1) !]^{2}} A^{2 d}=\frac{(2 d+2) !}{\pi^{d} 2^{d+1}[(d+1) !]^{2}} P^{d} .
$$

When $u \sim 1$, the nonlinearity has a strong effect and we have to resort to numerical methods to find the solution. We also notice that Eq. (13) with the Neumann boundary condition admits constant solution, i.e. $u=A_{0}$ with $E=A_{0}^{2 d} \geq 0$.

In Fig. 2, we solve Eq. (13) by using a shooting method to construct stationary modes for both Dirichlet and Neumann boundary conditions and find authentic law for $P(E)$. The stationary solutions $u(x)$ for $d=2$ are shown. The initial conditions are taken by the solutions (18) and
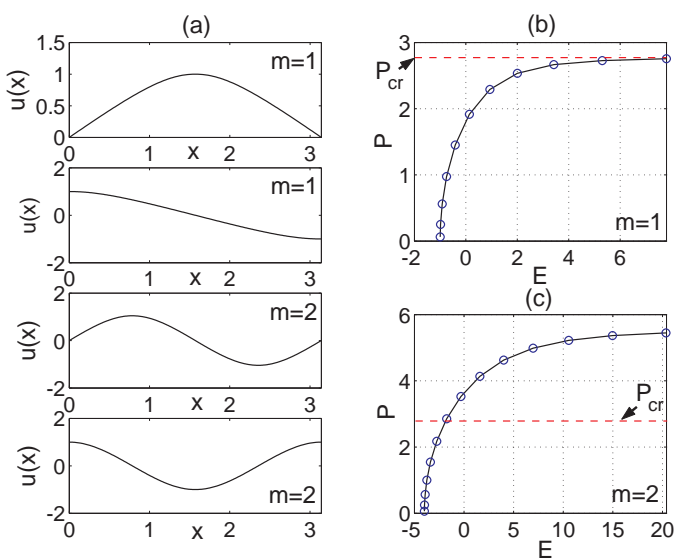

FIG. 2: The stationary solutions $u(x)$ for $d=2$. The initial conditions are taken as $\sin (m x)$ and $\cos (m x)$ naturally satisfying the Dirichlet and Neumann boundary conditions, respectively, with $m=1$ [the upper two panels in (a)] and $m=2$ [the lower two panels in (a)]. The dependence of $P$ on $E$ are shown in (b) and (c) for $m=1$ and $m=2$, respectively. The total power $\left.P\right|_{E \rightarrow \infty} \rightarrow 2.755$ for $m=1$ and $\left.P\right|_{E \rightarrow \infty} \rightarrow 5.447$ for $m=2$. The red line denotes the value of $P_{c r}$ by (25).

(19). We see that the family of solutions with $m=1$ bifurcates from zero at $E=E_{1}=-1$ while the family of solutions with $m=2$ bifurcates from zero at $E=E_{2}=-4$. Close to the bifurcation points the $P-E$ curves follow the relation (21). $P(E)$ is monotonically increasing with the growth of $E$ while $d P(E) /\left.d E\right|_{E \rightarrow \infty} \rightarrow 0$.

The linear stability of stationary solutions can be numerically computed by solving the linearized eigenvalue problem. Particularly, we assume that

$$
\psi(x, t)=[u(x)+(\alpha+i \beta)] e^{i E t},
$$

where $\alpha, \beta$ are small perturbations which are proportional to $\exp (-i \lambda t)$. The coupled eigenvalue problem then reads

$$
L_{1} B=-i \lambda A, \quad L_{2} A=i \lambda B
$$

where $L_{1}=-\partial_{x x}+E-u(x)^{2 d}$ and $L_{2}=-\partial_{x x}+E-$ $(2 d+1) u(x)^{2 d}$. The growth rate is defined as $\max [\operatorname{Im}(\lambda)]$ at which an unstable solution will grow. We find that the family of solutions with $m=1$ is always stable against linear perturbations whereas only a very narrow stability region close to the bifurcation point exists for the family of solutions with $m=2,3,4 \cdots$.

In the case of infinite domain, Eq. (13) admits the localized soliton solution

$$
u=[(d+1) E]^{1 / 2 d} \operatorname{sech}^{1 / d}\left[d \sqrt{E}\left(x-\frac{\pi}{2}\right)\right],
$$

where the solution is centered in the center of the domain $x=\pi / 2$. The total power for $d=2$ is a constant

$$
P_{c r}=\int|u|^{2} d x=\frac{\pi}{4} \sqrt{12}=2.72,
$$


i.e. it is independent on $E$. The solutions with power $P<P_{c r}$ disperse during the propagation, whereas if $P>P_{c r}$ the solutions collapse. A similar analysis can be carried out for the case of $d=3$ leading to

$$
P_{c r}=\int|u|^{2} d x=\frac{2^{5 / 3} \pi^{1 / 2} \Gamma(7 / 6)}{3^{1 / 2} \Gamma(2 / 3)} \frac{1}{E^{1 / 6}},
$$

which is expressed in terms of $\Gamma$ functions and depends on $E$. Although the soliton solutions do not satisfy the NLS equation on a bounded domain with specific boundary conditions, they are particularly useful as limiting cases of solutions.

The solution (24) can be considered as good approximations for the solutions satisfying both Dirichlet and Neumann boundary conditions in the limit $E \rightarrow \infty$. This is because in this latter limit the maximum intensity increases as $E^{1 / 2}$ while the pulse width decreases as $E^{1 / 2}$. The narrowing of the pulse makes the soliton tails, as well as their derivatives, are almost zero on the boundaries. The critical power for collapse $P_{c l}$ does not change with the growth of $E$.

\section{THE NUMERICAL SIMULATIONS}

To understand the dynamics of the Fourier modes, we relied heavily on numerical solutions of the NLS equation on the interval $[0, \pi]$. The equation was solved using the split-step Fourier method where the linear part is advanced using the sine or cosine Fourier transforms respectively for Dirichlet and Neumann boundary conditions. The details of the numerical implementation are given in the Appendix A.

We first consider Dirichlet boundary condition. The solution can be expanded in sines as

$$
\psi(t, x)=\sum_{m=1}^{\infty} c_{m}(t) \sin m x .
$$

For $d=1$, there is no collapse for Eq. (11). As expected the evolution of a sine initial condition gives rise to a cascade of modes. For $c_{1}(0)=2$ and $c_{j \neq 1}(0)=0$ we observe a cascade to $c_{3}$ and $c_{5}$ with maximum amplitudes $\max \left(c_{3}\right)=0.7$ and $\max \left(c_{5}\right)=0.15$ with the other modes being insignificant. For $c_{3}(0)=2$ and $c_{j \neq 3}(0)=0$ we get almost no cascade.

Now let us compare the outcomes for $d=1$ with the initial conditions $\psi(0, x)=\sin (x)+2 \sin (3 x)$ and $\psi(0, x)=\sin (x)+\sin (3 x)$. The time evolution of the mode amplitudes are shown in Fig. 3. The mode amplitudes fluctuate in a fairly narrow range around an average value. This range decreases even more for smaller initial amplitudes as shown in the right panel. We will explain these effects in the next section.

Let us now turn to the quintic case $(d=2)$ with Dirichlet boundary condition. In Fig. 4, we show the time evolution of solution (18) with $m=1$ and different values of amplitude $A$. No collapse is observed when
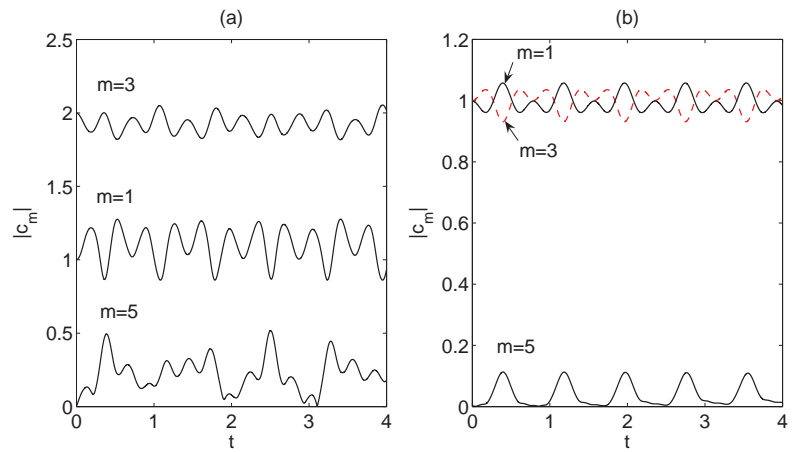

FIG. 3: Time evolution for a cubic nonlinearity and Dirichlet boundary conditions of the sine mode amplitudes $c_{m}(t), m=1,3,5$ starting from two different initial conditions $\psi(0, x)=\sin (x)+$ $2 \sin (3 x)$ (left panel) and $\psi(0, x)=\sin (x)+\sin (3 x)$ (right panel).

$A(P)$ is small whereas collapse occurs when $A>1.3$ $(P>2.65)$. A collapsing solution is shown on the last row of Fig. 4 for $A=1.31$. The right panels of Fig. 4 show the Fourier spectra. We notice that only the odd modes are excited. Actually, we can explain that the cascade of Fourier modes for Eq. (1) starting with a particular mode $q(q=0,1,2, \cdots)$ is always restricted to the modes $q(2 n-1)(n=1,2,3, \cdots)$ irrespective of the boundary conditions. In other words, we can expand the solutions of Eq. (11) as

$$
\psi(t, x)=\sum_{n=1}^{\infty} c_{q(2 n-1)} \sin [q(2 n-1) x]
$$

for the initial condition $\psi(0, x)=\sin (q x)$. For the Neumann boundary conditions the sines should be changed to cosines. Details are given in Appendix B. In Fig. 5, we show the recurrence of the solutions and spectrum cascade at different times when no collapse occurs. We will explain this phenomenon in the next section. We also notice that for large propagation constant, the solutions appears in the form of the hyperbolic secant function.

In Fig. 6. we show the time evolution of the Fourier amplitudes for a initial condition (18) with $m=1$ and increasing amplitude $A$. Each panel calculated for a single amplitude corresponds to different regions of similar behaviors for the modes. When $0<A \leq 0.5$, there is only the mode $m=1$. The other modes are insignificant. When $0.5<A \leq 1.0$, we observe two modes $m=1$ and 3. When $1.0<A \leq 1.2$, we observe three modes $m=1$, 3,5 . When $1.2<A \leq 1.3$, four modes $m=1,3,5$, and 7 are observed. The larger the amplitude of the initial condition, the more modes are excited. When $A>1.3$ collapse occurs. This is a much faster mechanism than the recurrence. The energy travels very suddenly from the low frequency modes to the higher frequency modes.

For the Neumann boundary conditions, the zero mode plays a special role. For example, it does not give rise to a spectrum cascade. We will see below how it couples to the other modes. Apart from this, the simulation results for the Neumann boundary conditions are similar to the 

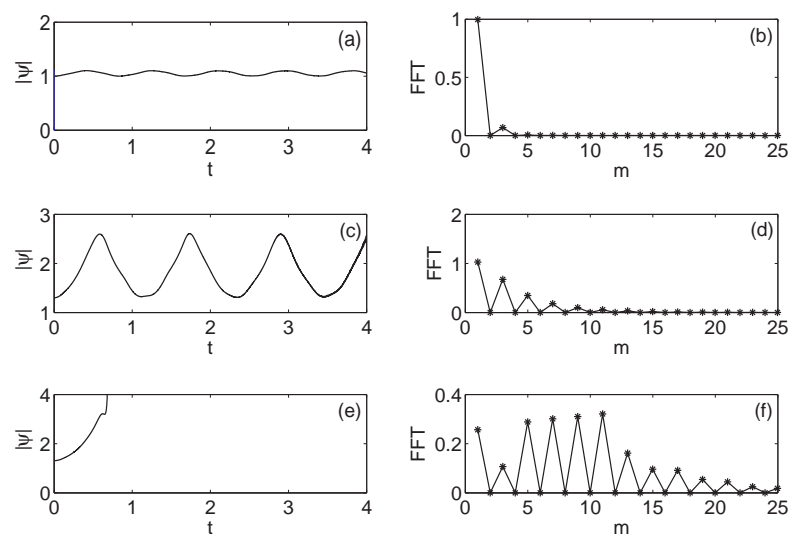

FIG. 4: Left panels: time evolution of the maximum of $|\psi|$ for a quintic nonlinearity and Dirichlet boundary conditions for three different initial conditions, from the top to the bottom, $\psi(0, x)=$ $1.0 \sin (x)(P=1.57), \psi(0, x)=1.3 \sin (x)(P=2.65)$ and $\psi(0, x)=$ $1.31 \sin (x)(P=2.70)$. The right panels show the corresponding Fourier spectra at time $t=4$. Notice the collapse occurring at $t \approx 0.7$ in the last row.
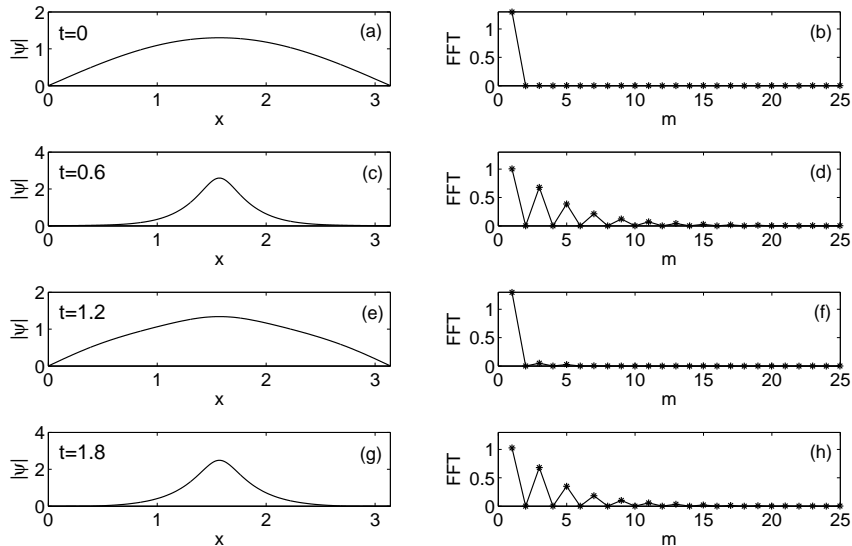

FIG. 5: Snapshots of the wave profiles and Fourier spectra for a quintic nonlinearity and Dirichlet boundary condition. The times shown are $t=0,0.6,1.2$ and 1.8 respectively in panels $\mathrm{a}, \mathrm{b}$, $\mathrm{c}$ and $\mathrm{d}$. The initial condition is $\psi(0)=1.3 \sin (x)(P=2.65)$. The recurrence of the wave profile and the spectrum cascade is evident. The profiles shown in (c) and (g) follow the hyperbolic secant function (24).

ones for the Dirichlet boundary conditions.

\section{EVOLUTION OF THE RESONANT FOURIER MODES}

In the interval of existence of solutions, one can use the Fourier sine or cosine series depending whether we have Dirichlet or Neumann boundary conditions. In both cases we have a complete basis for functions satisfying the boundary conditions. We will first consider the Dirichlet boundary condition and examine cubic and quintic nonlinearities. The results that we will obtain for these are very similar for the Neumann boundary condition un-
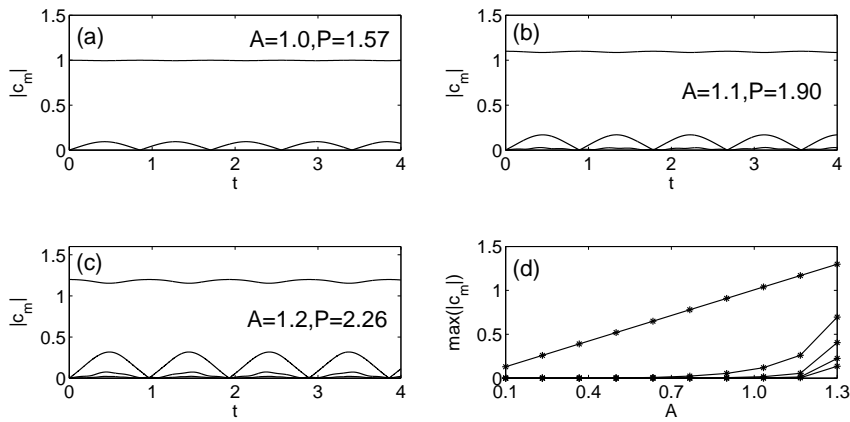

FIG. 6: Time evolution of the Fourier amplitudes for quintic nonlinearity and Dirichlet boundary condition for different initial conditions. In panels $\mathrm{a}, \mathrm{b}$ and $\mathrm{c}, c_{1}(0)=A=1$., 1.1 and 1.2 , respectively. The panel $\mathrm{d}$ shows $\max \left(\left|c_{m}\right|\right)$ for $m=1,3,5,7$ and 9 as a function of the initial amplitude $A$.

less the zero mode is involved. This specific case will be addressed at the end of the section.

For the Dirichlet boundary conditions, we expand $\psi$ in a sine Fourier series

$$
\psi(x, t)=\sum_{m=1}^{\infty} c_{m}(t) \sin (m x)
$$

where the $c_{m}$ are given by

$$
c_{m}(t)=\frac{2}{\pi} \int_{0}^{\pi} \psi(x, t) \sin (n x) d x .
$$

We find that if collapse occurs, it happens due to the divergence of the Fourier series. Indeed, the conservation of the power implies for any $m^{\prime}$

$$
\left|c_{m^{\prime}}(t)\right|^{2} \leq \sum_{m=1}^{\infty}\left|c_{m}(t)\right|^{2}=\frac{2}{\pi} P \equiv \mathcal{P}
$$

because of Parseval's relation,

$$
\int_{0}^{\pi}|\psi|^{2} d x=\frac{\pi}{2} \mathcal{P}
$$

Therefore the Fourier coefficients are bounded and the only source of collapse is the divergence of the series.

Substituting the expansion (29) into Eq. (11), we obtain the coupled equations of Fourier amplitudes. For the quintic nonlinearity $(d=2)$ we get

$$
\begin{aligned}
& i \dot{c}_{q}-q^{2} c_{q}+\frac{2}{\pi} \sum_{k, l, m, n, p} c_{k} c_{l} c_{m} c_{n}^{*} c_{p}^{*}\langle k l m n p \mid q\rangle \\
& =0
\end{aligned}
$$

where

$$
\begin{aligned}
\langle k l m n p \mid q\rangle \equiv & \int_{0}^{\pi} \sin (k x) \sin (l x) \sin (m x) \sin (n x) \\
& \times \sin (p x) \sin (q x) d x .
\end{aligned}
$$


Eqs. (32) can be simplified by the transformation $c_{q}=$ $a_{q} e^{-i q^{2} t}$ yielding

$$
\begin{gathered}
i \dot{a}_{q}+\frac{2}{\pi} \sum_{k, l, m, n, p} a_{k} a_{l} a_{m} a_{n}^{*} a_{p}^{*}\langle k \operatorname{lmn} n \mid q\rangle \\
\times e^{-i\left(k^{2}+l^{2}+m^{2}-n^{2}-p^{2}-q^{2}\right) t}=0 .
\end{gathered}
$$

Similarly, the coupled equations of Fourier amplitudes for the case of septic nonlinearity $(d=3)$ read

$$
\begin{aligned}
i \dot{a}_{s} & +\frac{2}{\pi} \sum_{k, l, m, n, p, q, r} a_{k} a_{l} a_{m} a_{n} a_{p}^{*} a_{q}^{*} a_{r}^{*}\langle k \text { lmnpqr } \mid s\rangle \\
& \times e^{-i\left(k^{2}+l^{2}+m^{2}+n^{2}-p^{2}-q^{2}-r^{2}-s^{2}\right) t}=0 .
\end{aligned}
$$

Note that in Eqs. (33) and (34) most terms are rapidly rotating and average out to zero. Only the ones such that $k^{2}+l^{2}+m^{2}-n^{2}-p^{2}-q^{2}=0$ for (33) $\left(k^{2}+l^{2}+\right.$ $m^{2}+n^{2}-p^{2}-q^{2}-r^{2}-s^{2}=0$ for (34)) i.e., the resonant terms will contribute to the long term dynamics of $a_{q}$.

A detailed study can be carried out of the dynamics of the Fourier coefficients for different types of nonlinearities. We have used the Maple software 8] to identify all the resonant terms in the equations for the mode amplitudes. For the cubic nonlinearity $(d=1)$ the amplitude equation equivalent to (33) reads

$$
\begin{gathered}
i \dot{a}_{j}+\frac{2}{\pi} \sum_{k, l, m} a_{k} a_{l} a_{m}^{*}\langle k l m \mid j\rangle \\
\times e^{-i\left(k^{2}+l^{2}-m^{2}-j^{2}\right) t}=0 .
\end{gathered}
$$

Taking into account the resonance condition, Eq. (35) turns into the following equations

$$
i \dot{a}_{j}+a_{j}\left(\mathcal{P}-\frac{\left|a_{j}\right|^{2}}{4}\right)=0, \quad(j=1,2, \cdots \infty)
$$

where $\mathcal{P}=\sum_{j=1}^{\infty}\left|a_{j}\right|^{2}$ is conserved. Eq. (36) admits the solution

$$
a_{j}=\left|a_{j}\right| e^{i\left(\mathcal{P}-\left|a_{j}\right|^{2} / 4\right) t} .
$$

An obvious implication is that $d / d t\left(\left|a_{j}\right|^{2}\right)=0$ so that there is no transfer of energy from one mode to another. This is what we have seen in the numerical results in Fig. 3. Over a short time the modes oscillate in a periodic fashion, however their average over a long time is constant. As expected no collapse will occur in the cubic NLS equation irrespective of the initial total power. In fact the integrability of the NLS equation on the whole line, related to the existence of a Lax pair, has been shown to carry over to the case of a finite domain with Dirichlet boundary conditions [9].

The case of the quintic nonlinearity $(d=2)$ is more complicated. For simplicity, we consider a solution consisting of three modes, i.e., $m=1,3$ and 5 ,

$$
\begin{aligned}
\psi(t, x) & =a_{1} e^{-i t} \cos (x)+a_{3} e^{-i 9 t} \cos (3 x) \\
& +a_{5} e^{-i 25 t} \cos (5 x) .
\end{aligned}
$$

Then Eq. (33) gives rise to the following coupled resonant amplitude equations

$$
\begin{gathered}
i \dot{a}_{1}+a_{1}\left[\frac{9}{4}\left|a_{1}\right|^{2} \mathcal{P}-\frac{13}{8}\left|a_{1}\right|^{4}+3\left|a_{3}\right|^{2}\left|a_{5}\right|^{2}\right. \\
\left.+\frac{9}{8}\left(\left|a_{3}\right|^{4}+\left|a_{5}\right|^{4}\right)\right]-\frac{3}{8} a_{1}^{*} a_{3}^{3} a_{5}^{*}=0, \\
i \dot{a}_{3}+a_{3}\left[\frac{9}{4}\left|a_{3}\right|^{2} \mathcal{P}-\frac{13}{8}\left|a_{3}\right|^{4}+3\left|a_{1}\right|^{2}\left|a_{5}\right|^{2}\right. \\
\left.+\frac{9}{8}\left(\left|a_{1}\right|^{4}+\left|a_{5}\right|^{4}\right)\right]-\frac{9}{16} a_{1}^{2} a_{3}^{* 2} a_{5}=0, \\
i \dot{a}_{5}+a_{5}\left[\frac{9}{4}\left|a_{5}\right|^{2} \mathcal{P}-\frac{13}{8}\left|a_{5}\right|^{4}+3\left|a_{1}\right|^{2}\left|a_{3}\right|^{2}\right. \\
\left.\quad+\frac{9}{8}\left(\left|a_{1}\right|^{4}+\left|a_{3}\right|^{4}\right)\right]-\frac{3}{16} a_{1}^{* 2} a_{3}^{3}=0,
\end{gathered}
$$

where $\mathcal{P}=\left|a_{1}\right|^{2}+\left|a_{3}\right|^{2}+\left|a_{5}\right|^{2}$. It is easy to check that Eqs. (39) satisfy the condition $\frac{d}{d t}\left(\left|a_{1}\right|^{2}+\left|a_{3}\right|^{2}+\left|a_{5}\right|^{2}\right)=0$, i.e., $\mathcal{P}$ is a conserved quantity. The last terms in equations represent the mixing between the three modes and therefore the intensity of each mode is not conserved as in the case of $d=1$. At this point note that if we had included $a_{7}$ in the description we would have had the additional resonant terms

$$
\left|a_{3}\right|^{2} a_{5}^{2} a_{7}, \quad a_{5}^{2}\left|a_{7}\right|^{2} a_{7}^{*}, \quad a_{5}^{2}\left|a_{5}\right|^{2} a_{7}^{*} .
$$

If $\left|a_{7}\right|<<1$ then their contribution would be very small. Another point is that if we had included the even modes in the description we would have obtained the extra resonant term $a_{2}^{2} a_{4}^{* 2} a_{5}$ in the equation for $a_{1}$. This indicates that the modes 2 and 4 couple through mode 5 .

Eqs. (39) can be written into a more compact form by defining $I_{j}=\left|a_{j}\right|^{2}(j=1,3,5)$. Here, $I_{j}$ represent the intensity of each mode satisfying $\mathcal{P}=I_{1}+I_{3}+I_{5}$. Then, we obtain

$$
\begin{aligned}
& \dot{I}_{1}=\frac{3}{4} I_{1} I_{3}^{3 / 2} I_{5}^{1 / 2} \sin \theta, \\
& \dot{I}_{3}=-\frac{9}{8} I_{1} I_{3}^{3 / 2} I_{5}^{1 / 2} \sin \theta, \\
& \dot{I}_{5}=\frac{3}{8} I_{1} I_{3}^{3 / 2} I_{5}^{1 / 2} \sin \theta,
\end{aligned}
$$

where $\theta=-2 \theta_{1}+3 \theta_{3}-\theta_{5}$ with $\theta_{j}=\arg a_{j}$. We notice that the driving terms on the right hand side of Eqs. (40) are proportional to the intensities of the modes and their phases. In addition, we have the constraints

$$
\begin{aligned}
& \frac{4}{3} I_{1}+\frac{8}{9} I_{3}=\mu_{1}, \\
& \frac{8}{9} I_{3}+\frac{8}{3} I_{5}=\mu_{2}, \\
& \frac{4}{3} I_{1}-\frac{8}{3} I_{5}=\mu_{1}-\mu_{2}=\mu_{3},
\end{aligned}
$$

where $\mu_{i},(i=1-3)$ are constants of the motion. Using the above relations, the dynamics can be reduced to the 
equations for $I_{1}$ and $\theta$. They are

$$
\begin{aligned}
\dot{I}_{1} & =\frac{3}{4} I_{1} I_{3}^{3 / 2} I_{5}^{1 / 2} \sin \theta \\
\dot{\theta} & =\frac{1}{4}\left(13 I_{1}^{2}+3 I_{3}^{2}+7 I_{5}^{2}+21 I_{1} I_{3}+27 I_{1} I_{5}+12 I_{3} I_{5}\right) \\
& +\frac{\cos \theta}{8}\left[6 I_{1}^{1 / 2} I_{3}^{3 / 2} I_{5}^{1 / 2}-27 I_{1} I_{3} I_{5}^{1 / 2}\right. \\
& \left.-3 I_{1} I_{3}^{3 / 2} I_{5}^{-1 / 2}\right],
\end{aligned}
$$

together with the constraints (41).

In order to check the equations for the resonant Fourier modes, we compare the solutions of the reduced equations and the NLS. In Fig. 7 we show the time evolution of the solutions of Eqs. (40) and (11) by using the Runge-Kutta method and split-step Fourier method, respectively. The initial conditions are the same. As expected the amplitudes of the Fourier modes for (1) present fast periodic oscillations. However over a long time interval the solutions of the reduced equations and the full partial differential equation match well, supporting the validity of the reduced model.

At this point, let us consider the Neumann boundary conditions for which there is the additional zero mode. For the cubic nonlinearity $d=1$, the evolution of the Fourier modes follows (36) so that there is no resonant energy exchange between the modes $\dot{I}_{j}=0, j=0,1, \ldots$. For the quintic nonlinearity $d=2$, the situation is more interesting. Assuming a solution containing the three modes $i=1,3,5$, we obtain evolution equations identical to (39) except that the signs of the resonant terms are reversed. We then obtain the same final equations (42) except that the evolution of the phase $\theta$ is reversed.

Assuming a solution containing the three first modes $i=0,1,2$, we obtain evolution equations of the form (39) but with no terms outside the brackets. This means that again, no resonant transfer of energy exists between modes. If the third mode is added to the expansion, new terms appear outside the brackets. The evolution equations are

$$
\begin{aligned}
& i \dot{a}_{0}+a_{0}[\ldots]+\frac{3}{4} a_{0}^{*} a_{1} a_{2}^{2} a_{3}^{*}=0, \\
& i \dot{a}_{1}+a_{1}[\ldots]+\frac{3}{4} a_{0}^{2} a_{2}^{* 2} a_{3}=0 \\
& i \dot{a}_{2}+a_{2}[\ldots]+\frac{3}{2} a_{0}^{2} a_{1}^{*} a_{2}^{*} a_{3}=0, \\
& i \dot{a}_{3}+a_{3}[\ldots]+\frac{3}{4} a_{0}^{* 2} a_{1} a_{2}^{2}=0,
\end{aligned}
$$

where the ... terms in the brackets are all real. Following a similar procedure as above, the modal energies $I_{j}=$

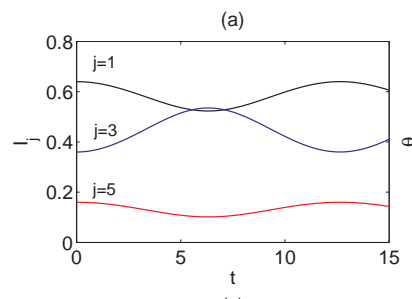

(c)
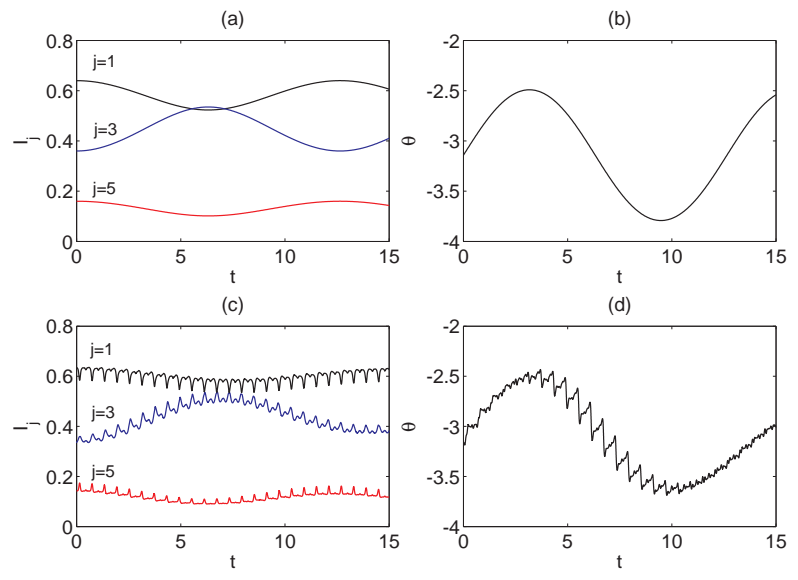

(d)

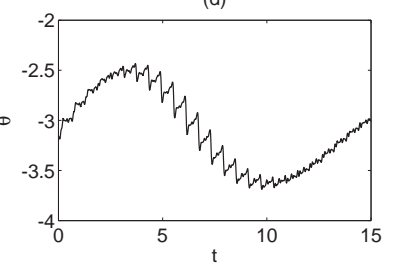

FIG. 7: Comparison between the solution of the reduced model and the numerical solution of the NLS for quintic nonlinearity and Dirichlet boundary condition. The top left (right) panel shows the time evolution $I_{1}(t), I_{3}(t)$, and $I_{5}(t)(\theta(t))$ for the reduced model (42). The bottom left (right) panel shows the corresponding evolutions for the NLS. The initial conditions are taken as $I_{1}=$ $0.64, I_{3}=0.36$ and $I_{5}=0.16$ and $\theta=-\pi$.

$\left|a_{j}\right|^{2}$ evolve as

$$
\begin{aligned}
\dot{I}_{0} & =\frac{3}{2} I_{0} I_{1}^{1 / 2} I_{2} I_{3}^{1 / 2} \sin \theta \\
\dot{I}_{1} & =\frac{3}{2} I_{0} I_{1}^{1 / 2} I_{2} I_{3}^{1 / 2} \sin \theta \\
\dot{I}_{2} & =3 I_{0} I_{1}^{1 / 2} I_{2} I_{3}^{1 / 2} \sin \theta \\
\dot{I}_{3} & =\frac{3}{2} I_{0} I_{1}^{1 / 2} I_{2} I_{3}^{1 / 2} \sin \theta
\end{aligned}
$$

where $\theta=-2 \theta_{0}+\theta_{1}+2 \theta_{2}-\theta_{3}$. As above one can then reduce the problem to two equations, one for $I_{0}$ and one for $\theta$. We do not write these equations because they are cumbersome. The interesting fact is that one needs the 4 modes 0-3 present in order to see this resonant transfer of energy. If one of them is missing there is no energy transfer. This particular feature of Neumann boundary conditions changes the route for collapse for the Dirichlet case and the Neumann case.

\section{FILTERING FOURIER MODES PREVENTS COLLAPSE}

The projection of the nonlinear Schrödinger equation on a a finite number of Fourier modes will yield amplitude equations that are well behaved and do not exhibit collapse. As we have seen collapse is related to a sudden energy flow to high frequencies. Thus, one can arrest the collapse by filtering the high Fourier modes and therefore preventing the sudden energy flow to high frequencies. Physically this can be done by introducing a nonlocal absorption in the model (11). This conclusion is also available for the models with septic nonlinearity and Neumann boundary condition. 


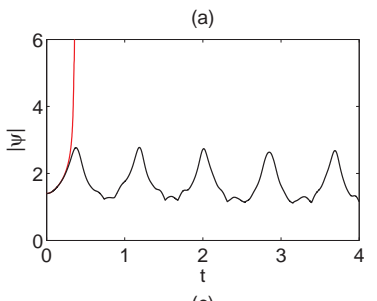

(c)

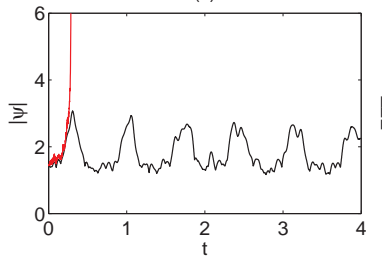

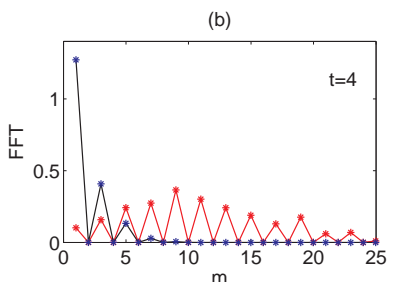

(d)

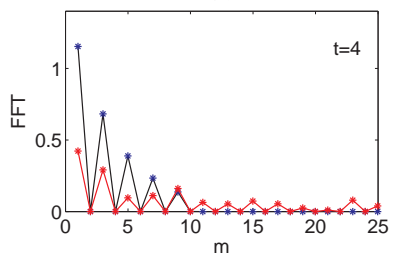

FIG. 8: Left panels: Time evolution of the maximum of $|\psi|$ for quintic nonlinearity with different initial conditions. Right panels: The corresponding Fourier spectra at $t=4$. The initial conditions are taken as $\psi(0, x)=1.4 \sin (x)(P(0)=3.08)$ in the first row and $\psi(0, x)=1.4 \operatorname{sech}\left(x-\frac{\pi}{2}\right)(P(0)=3.56)$ in the second row. We compare the results with and without filtering the high Fourier modes designated by the black and red lines, respectively. The collapse is efficiently prevented by filtering in both cases.

In Fig. 8 we show the time evolution of solution with or without filtering high Fourier modes. The initial conditions are taken as a sine wave (the first row) and a hyperbolic secant pulse (the second row). In both cases, the collapse is efficiently prevented by filtering. Because of the absorption of the higher modes, there is a small loss (less than 10 percent) of the total power. This can be decreased by including more lower frequency modes.

\section{CONCLUSION}

We have analyzed and solved numerically the one dimensional nonlinear Schrödinger equation on a finite interval with Dirichlet or Neumann boundary conditions. A preliminary analysis reveals that $H<0$ is sufficient for collapse in the Dirichlet case but not for the Neumann case. The bound states have been computed. The first non trivial one corresponding in the linear limit to $\sin x($ resp. $\cos x)$ for Dirichlet (resp. Neumann) boundary conditions is always linearly stable as opposed to the higher order modes whose window of stability is very small and reduces as the order is increased.

We have solved the partial differential equation for the cubic and quintic nonlinearities. In the cubic case there is no resonant transfer of energy between Fourier modes, while it is present in the quintic case. Identifying resonant terms in the evolution equations of the Fourier modes, we have written reduced systems. Their evolution is in excellent agreement with the solutions of the NLS, even close to collapse. For the Neumann boundary conditions the Goldstone mode plays a particular role as it couples the modes $m=1,2,3$. For the Dirichlet case, there is no coupling between the first three modes. Fi- nally note that this model reduction can be extended to higher dimensions and other systems like a cylindrical waveguide. The machinery would be more complicated but the overall method remains the same.

\section{Acknowledgments}

The research of JGC and $\mathrm{CH}$ was supported by a joint France-Portugal Pessoa agreement. The research of $\mathrm{CH}$ was supported by the Fundação para a Ciência e a Tecnologia (FCT) under Grant No. SFRH/BPD/36385/2007 and Estímulo à Investigação 2009 de Fundação Calouste Gulbenkian. The authors thank Vladimir Konotop for very helpful discussions.

\section{Appendix A: Numerical procedure for solving the 1D NLS (1)}

The 1D nonlinear Schrödinger equation (1) with Dirichlet or Neumann boundary conditions is solved as usual by splitting the linear and nonlinear part of the operator. The linear part

$$
i u_{t}+u_{x x}=0,
$$

is such that

$$
\hat{u}(d t)=e^{-i k^{2} d t} \hat{u}(0),
$$

where $\hat{u}$ is the sine or cosine Fourier transform of $u$, to satisfy the boundary conditions. The solution of the nonlinear stepping is the standard one

$$
u(2 d t)=e^{i|u(d t)|^{2 d} d t} u(d t) .
$$

The numerical implementation is done in Matlab [7] and the solution is evaluated at discrete points $u_{n}, n=$ $1, \ldots N$. The sine and cosine Fourier transforms for the linear step (A1) are then done using the discrete sine and cosine Fourier transforms.

For the Dirichlet boundary conditions, we use the discrete sine Fourier transform

$$
\hat{u}(k)=\sum_{n=1}^{N} u(n) \sin \left(\frac{\pi k n}{N+1}\right), \quad k=1, \ldots N,
$$

and inverse discrete sine Fourier transform

$$
u(n)=\sum_{k=1}^{N} \hat{u}(k) \sin \left(\frac{\pi k n}{N+1}\right), \quad n=1, \ldots N .
$$

The Neumann boundary conditions are trickier to implement because one needs to use the discrete cosine Fourier transform

$$
\hat{u}(k)=w(k) \sum_{n=1}^{N} u(n) \cos \left(\frac{\pi(2 n-1)(k-1)}{2 N}\right), k=1, \ldots N,
$$


with

$$
w(1)=1 / \sqrt{N}, \quad w(k)=\sqrt{\frac{2}{N}}, \quad 2 \leq k \leq N
$$

. The inverse discrete cosine Fourier transform is

$u(n)=\sum_{k=1}^{N} w(k) \hat{u}(k) \cos \left(\frac{\pi(2 n-1)(k-1)}{2 N}\right), n=1, \ldots N$.

The number of discretisation points was chosen to be $N=2^{11}-1$ or $N=2^{12}-1$ with a step $d t=10^{-4}$. The $\mathrm{L}_{2}$ norm was checked during the computation and it is conserved up to $10^{-10}$ in absolute value.

\section{Appendix B: Parity argument for the mode cascade}

In order to prove Eq. (28), let us consider the case of $d=1$, the cases of $d=2,3$ can be proved in a similar way. Eq. (11) with $d=1$ can be written into the form

$$
\begin{aligned}
& \psi(t+d t, x)=\psi(t, x)+i\left[\frac{1}{2} \frac{\partial^{2} \psi(t, x)}{\partial x^{2}}\right. \\
& \left.+|\psi(t, x)|^{2} \psi(t, x)\right] d t .
\end{aligned}
$$

Now we assume that the initial condition is taken as $\psi(0, x)=\cos (q x)=\frac{1}{2}\left(e^{i q x}+e^{-i q x}\right)$. Substituting the initial condition into Eq. (B1), we have

$$
\begin{aligned}
& \psi(d t, x)=\frac{1}{2}\left(e^{i q x}+e^{-i q x}\right)+i\left[-\frac{1}{4}\left(e^{i q x}+e^{-i q x}\right)\right. \\
& \left.+\frac{1}{8}\left(e^{3 i q x}+3 e^{i q x}+3 e^{-i q x}+e^{-3 i q x}\right)\right] d t .
\end{aligned}
$$

As we see, after a short time interval $d t$, the solution $\psi(d t, x)$ can be expressed as $\psi(d t, x)=c_{q} \cos (q x)+$ $c_{3 q} \cos (3 q x)$. We can repeat this process and obtain that $\psi(t, x)=\sum_{m=(2 n-1) q} c_{m} \cos (m x) \quad(n=1,2, \cdots)$. In other words for $d=1$ we immediately obtain modes 1 , 3 and 5 while for $d=2$ we immediately obtain modes 1 , 3,5 and 7, etc. Finally, notice that for Neumann boundary condition there is no cascade to higher Fourier modes starting with the $m=0$ mode.

\section{Appendix C: Evolution of the resonant Fourier modes for the Neumann boundary conditions}

For the Neumann case, we expand $\psi$ in a cosine Fourier series

$$
\psi(x, t)=\sum_{m=0}^{\infty} c_{m}(t) \cos (m x),
$$

where the $c_{m}$ are given by

$$
\begin{aligned}
& c_{0}(t)=\frac{1}{\pi} \int_{0}^{\pi} \psi(x, t) d x \\
& c_{n}(t)=\frac{2}{\pi} \int_{0}^{\pi} \psi(x, t) \cos (n x) d x \quad(n \neq 0) .
\end{aligned}
$$

Substituting the expansion (C1) into Eq. (1), we obtain the coupled equations of Fourier amplitudes for the case of quintic nonlinearity $(d=2)$

$$
\begin{aligned}
& i \dot{c}_{0}+\frac{1}{\pi} \sum_{k, l, m, n, p} c_{k} c_{l} c_{m} c_{n}^{*} c_{p}^{*}\langle k \operatorname{lmn} p \mid 0\rangle=0, \quad(\mathrm{C} 3 \mathrm{r} \\
& i \dot{c}_{q}-q^{2} c_{q}+\frac{2}{\pi} \sum_{k, l, m, n, p} c_{k} c_{l} c_{m} c_{n}^{*} c_{p}^{*}\langle k l m n p \mid q\rangle \\
& =0, \quad(q \neq 0)
\end{aligned}
$$

where

$$
\begin{aligned}
\langle k l m n p \mid q\rangle \equiv & \int_{0}^{\pi} \cos (k x) \cos (l x) \cos (m x) \cos (n x) \\
& \times \cos (p x) \cos (q x) d x .
\end{aligned}
$$

Eqs. (C3) can be simplified by the transformation $c_{q}=$ $a_{q} e^{-i q^{2} t}$ yielding

$$
\begin{gathered}
i \dot{a}_{q}+\frac{\sigma_{q}}{\pi} \sum_{k, l, m, n, p} a_{k} a_{l} a_{m} a_{n}^{*} a_{p}^{*}\langle k \operatorname{lmn} n \mid q\rangle \\
\times e^{-i\left(k^{2}+l^{2}+m^{2}-n^{2}-p^{2}-q^{2}\right) t}=0,
\end{gathered}
$$

where $\sigma_{q}=1$ for $q=0$ and $\sigma_{q}=2$ for $q \neq 0$. Similarly, the coupled equations of Fourier amplitudes for the case of septic nonlinearity $(d=3)$ read

$$
\begin{aligned}
& i \dot{a}_{s}+\frac{\sigma_{s}}{\pi} \sum_{k, l, m, n, p, q} a_{k} a_{l} a_{m} a_{n} a_{p}^{*} a_{q}^{*} a_{r}^{*}\langle k l m n p q r \mid s\rangle \\
& \quad \times e^{-i \frac{1}{2}\left(k^{2}+l^{2}+m^{2}+n^{2}-p^{2}-q^{2}-r^{2}-s^{2}\right) t}=0
\end{aligned}
$$

where $\sigma_{s}=1$ for $s=0$ and $\sigma_{s}=2$ for $s \neq 0$.

For simplicity, we consider the solution (38). Then, Eq. (C4) turns into the following coupled equations

$$
\begin{gathered}
i \dot{a}_{1}+a_{1}\left[\frac{9}{4}\left|a_{1}\right|^{2} P-\frac{13}{8}\left|a_{1}\right|^{4}+3\left|a_{3}\right|^{2}\left|a_{5}\right|^{2}\right. \\
\left.+\frac{9}{8}\left(\left|a_{3}\right|^{4}+\left|a_{5}\right|^{4}\right)\right]+\frac{3}{8} a_{1}^{*} a_{3}^{3} a_{5}^{*}=0, \\
i \dot{a}_{3}+a_{3}\left[\frac{9}{4}\left|a_{3}\right|^{2} P-\frac{13}{8}\left|a_{3}\right|^{4}+3\left|a_{1}\right|^{2}\left|a_{5}\right|^{2}\right. \\
\left.+\frac{9}{8}\left(\left|a_{1}\right|^{4}+\left|a_{5}\right|^{4}\right)\right]+\frac{9}{16} a_{1}^{2} a_{3}^{* 2} a_{5}=0, \\
i \dot{a}_{5}+a_{5}\left[\frac{9}{4}\left|a_{5}\right|^{2} P-\frac{13}{8}\left|a_{5}\right|^{4}+3\left|a_{1}\right|^{2}\left|a_{3}\right|^{2}\right. \\
\left.\quad+\frac{9}{8}\left(\left|a_{1}\right|^{4}+\left|a_{3}\right|^{4}\right)\right]+\frac{3}{16} a_{1}^{* 2} a_{3}^{3}=0,
\end{gathered}
$$

where $\mathcal{P}=\left|a_{1}\right|^{2}+\left|a_{3}\right|^{2}+\left|a_{5}\right|^{2}$. These are exactly the same as (39) except that the terms outside the brackets have the opposite signs.

As done above, eqs. (C6) can be written into a more 
compact form by defining $I_{j}=\left|a_{j}\right|^{2}(j=1,3,5)$, i.e.

$$
\begin{aligned}
& \dot{I}_{1}=-\frac{3}{4} I_{1} I_{3}^{3 / 2} I_{5}^{1 / 2} \sin \theta \\
& \dot{I}_{3}=\frac{9}{8} I_{1} I_{3}^{3 / 2} I_{5}^{1 / 2} \sin \theta \\
& \dot{I}_{5}=-\frac{3}{8} I_{1} I_{3}^{3 / 2} I_{5}^{1 / 2} \sin \theta
\end{aligned}
$$

where $\theta=-2 \theta_{1}+3 \theta_{3}-\theta_{5}$ with $\theta_{j}=\arg a_{j}$. Eq. (C7) can be further written into the form

$$
\begin{aligned}
\dot{I}_{1} & =-\frac{3}{4} I_{1} I_{3}^{3 / 2} I_{5}^{1 / 2} \sin \theta, \quad(\mathrm{C} 8 \mathrm{a}) \\
\dot{\theta} & =\frac{1}{4}\left(13 I_{1}^{2}+3 I_{3}^{2}+7 I_{5}^{2}+21 I_{1} I_{3}+27 I_{1} I_{5}+12 I_{3} I_{5}\right) \\
& -\frac{\cos \theta}{8}\left[6 I_{1}^{1 / 2} I_{3}^{3 / 2} I_{5}^{1 / 2}-27 I_{1} I_{3} I_{5}^{1 / 2}\right. \\
& \left.-3 I_{1} I_{3}^{3 / 2} I_{5}^{-1 / 2}\right],
\end{aligned}
$$

together with the constraints (41).
[1] Luc Bergé, Phys. Rep. 303 259(1998).

[2] C. Sulem and P.-L. Sulem, The nonlinear Schrödinger equation, Springer, N. Y. , (1999).

[3] G. Fibich and F. Merle, Physica D 155, 132-158 (2001).

[4] "Nonlinear waves in complex systems: energy flow and geometry", J. G. Caputo and M. P. Soerensen Eds. , "Special Topics" of European J. Phys. and Springer, (2007).

[5] D. J. Muraki, SIAM Journal on Applied Mathematics, (2007).
[6] V. Zakharov, F. Dias and A. Pushkarev, Phys. Reports 398,1-65, (2004).

[7] The MathWorks http://www.mathworks.com

[8] http://www.maplesoft.com/

[9] A. S. Fokas and A. R. Its, The nonlinear Schrödinger on the interval J. Phys. A: Math. Gen. 37 (2004) 60916114. 